OPEN ACCESS

Citation: A.C. Baukloh. (2021) Conflict capability e mediazione: un approccio preventivo alla radicalizzazione e ai comportamenti antisociali in contesti familiari e microsociali. Rief 18 , 2: pp. 91-106. doi: https://doi. org/10.36253/rief-10558.

Copyright: (c) 2021 A. C. Baukloh. This is an open access, peer-reviewed article published by Firenze University Press (https://oaj.fupress.net/index. php/rief) and distributed under the terms of the Creative Commons Attribution License, which permits unrestricted use, distribution, and reproduction in any medium, provided the original author and source are credited.

Data Availability Statement: All relevant data are within the paper and its Supporting Information files.

Competing Interests: The Author(s) declare(s) no conflict of interest.

\section{Conflict capability e mediazione: un approccio preventivo alla radicalizzazione e ai comportamenti antisociali in contesti familiari e microsociali}

\author{
Anja Corinne Baukloh ${ }^{1}$
}

\section{Abstract}

L'analisi delle dinamiche dei conflitti tra gli individui e nella società e l'elaborazione di appropriate strategie di intervento offrono una prospettiva importante per la prevenzione dei processi di radicalizzazione. Larticolo fornisce una panoramica di alcuni strumenti concreti di analisi e intervento nelle dinamiche di conflitto microsociale, con particolare riferimento alla famiglia. Il punto di partenza scelto è il concetto di "conflict capability", la capacità degli esseri umani di gestire in modo appropriato un conflitto. L'approccio presentato unisce uno sguardo sistemico al fenomeno e un accompagnamento trasformativo alle capacità delle singole persone, in particolare delle donne, di affrontare i conflitti e cercare una soluzione. Il modello di analisi di Friedrich Glasl dei processi di escalation conflittuale articolato in nove stadi rappresenta un importante riferimento per definire la dinamica del conflitto concreto e il tipo di intervento. Gli strumenti di intervento nel contesto familiare e microsociale proposti nel testo sono il modello di comunicazione empatica e "problem solving senza perdenti" elaborato da Thomas Gordon e l'approccio della mediazione sistemica trasformativa dei conflitti. L'articolo propone infine una riflessione sull'importanza di una cultura diffusa della gestione costruttiva dei conflitti - la conflict capability - per rafforzare la pratica della democrazia nelle società contemporanee, con riferimento ad Autori come Chantal Mouffe e Axel Honneth.

Parole chiave: conflitti, mediazione, radicalizzazione, educazione, cambiamento.

Abstract

Understanding conflict dynamics among individuals and within society, and devising appropriate intervention strategies offer a relevant perspective for the prevention of radicalization processes. The article outlines an overview of some

\footnotetext{
${ }^{1}$ Responsabile operativa del Laboratorio "FORMA MENTIS - Trasformazione dei Conflitti e Apprendimento Sistemico”, del PIN S.c.r.l. - Servizi didattici e scientifici per l'Università degli Studi di Firenze, e Ricercatrice aggregata del Centro Interdisciplinare Scienze per la Pace (CISP) dell'Università degli Studi di Pisa.
} 
concrete instruments of analysis and intervention in microsocial conflicts, with special reference to family. We choose as a starting point the notion of "conflict capability", the ability of human beings of appropriately handling conflict. The model presented here is based on a systemic understanding of social conflict and a transformative approach to foster human capabilities, and specifically women, of dealing with conflict. Friedrich Glasl's "escalation model" offers a solid foundation in order to define conflict dynamics which is crucial for devising appropriate, context-specific interventions. For the family and microsocial context, we propose as intervention tools empathic communication and "problem solving without losers" proposed by Thomas Gordon, as well as a "systemic transformative" approach to mediation. The article also reflects on the importance of conflict capability in order to reinforce the practice of democracy, building on the contribution of authors such as Chantal Mouffe and Axel Honneth.

Keywords: conflict, mediation, radicalization, education, change processes.

\section{Introduzione}

I conflitti fanno parte della famiglia come di qualsiasi sistema sociale, e il loro impatto dipende dal modo in cui vengono gestiti. In quanto agenzia di socializzazione primaria, decisiva per l'acquisizione dell'identità sociale (Ribolzi, 2012) la famiglia può diventare un contesto di apprendimento di attitudini positive verso la nonviolenza, un laboratorio di gestione costruttiva dei conflitti: in questo caso è assai probabile che i suoi membri saranno in grado di gestire meglio non solo i conflitti familiari, ma anche le tensioni interiori e i conflitti nell'ambiente sociale. Perciò riteniamo la capacità di comprendere e trasformare le dinamiche conflittuali un elemento importante per la prevenzione della radicalizzazione e dei comportamenti antisociali nel contesto familiare.

In questa prospettiva proponiamo qui alcuni elementi chiave della mediazione sistemica e trasformativa (Ballreich, Glasl, 2019, trad. it. 2021; Baukloh, 2008; Baukloh, Scotto, 2019; Baukloh, Valdambrini, 2015; Bush, Folger, 2004, trad.it. 2009; Glasl, 1997, trad. it. 2019; Panerai, Baukloh, 2014; Scotto, 2018) per l'analisi e l'intervento nelle dinamiche di conflitto familiare e microsociale. Si tratta di concetti teorici e strumenti pratici che possono dare un contributo importante allo sviluppo di atteggiamenti costruttivi di apertura e di dialogo, con l'obiettivo di sviluppare una maggiore conflict capability: la capacità degli esseri umani di gestire in modo appropriato un conflitto.

La competenza in materia di conflitti non va confusa con il loro evitamento: questo vale sia al livello famigliare che sociale. Un approccio costruttivo ai conflitti sociali può anche consistere nel dare piena voce a posizioni radicali, ma con forme di espressione che non siano espressione di violenza, e con la volontà di ascoltare le posizioni degli altri e di competere con loro in un confronto discorsivo.

A nostro avviso, coltivare la competenza a gestire in modo appropriato i conflitti nella sfera familiare e microsociale potrà costituire un punto di partenza per una società più resiliente $\mathrm{e}$ capace di affrontare conflitti e sfide del presente.

\section{Dalla radicalizzazione all'agonismo democratico}

Nel libro Il conflitto democratico, Chantal Mouffe fornisce per l'ambito della politica una riflessione analoga: 
Il conflitto non può e non deve essere sradicato dalle società democratiche liberali, dato che la specificità della democrazia pluralista risiede precisamente nel riconoscimento e nella legittimazione del conflitto. Una politica democratica liberale esige semmai che gli altri non siano intesi come nemici da distruggere, ma avversari le cui idee possono essere combattute, anche con ferocia, ma senza mettere mai in discussione il loro diritto a difenderle. Per dirla in altro modo, è importante che il conflitto non assuma la forma di un "antagonismo" (lotta tra nemici) bensì la forma di un "agonismo" (lotta tra avversari)" (2000, trad. it. 2015, pp. 26-27).

Per far sì che il sistema politico democratico funzioni, c’è bisogno di un confronto democratico vivace, che includa anche punti di vista e posizioni marginalizzate e permetta a tutti di esprimere il proprio dissenso, sulla base della condivisione dei valori etici e politici e la discussione su cosa significano questi valori e su come devono essere attuati nella pratica: «Il consenso, quindi, è sempre un "consenso conflittuale". La competizione agonistica offre ai cittadini diverse possibilità di identificazione, offrendo quindi uno sbocco democratico per le passioni politiche» (ivi, p. 28). Mentre il conflitto tra avversari, la "lotta agonistica", è la condizione fondamentale per l'esistenza di una democrazia vitale, la sua mancanza - constata Mouffe - rischia di portare a situazioni in cui «il confronto democratico sia sostituito da un confronto tra valori morali non negoziabili, o forme essenzialiste di identificazione» (ivi, p. 27).

Assumendo la prospettiva di Mouffe, possiamo intendere la radicalizzazione come un processo di sviluppo di atteggiamenti "antagonistici", che portano a vedere l'avversario come nemico e favoriscono l'escalation conflittuale fino a estremismi violenti, mentre l'educazione alla gestione costruttiva dei conflitti è associata allo sviluppo di un atteggiamento non violento e alla capacità di lottare per le proprie convinzioni in modo "agonistico", senza estremismi violenti.

\section{Conflict capability}

Affinché nella società i conflitti possano essere gestiti in modo non violento, vivace e appassionato, occorre esercitare le relative competenze e capacità. In quanto agenzie di socializzazione, scuola e famiglia svolgono un ruolo chiave in questo senso, non solo per quanto riguarda la generica diffusione dei valori democratici, ma anche per la conoscenza e la pratica delle modalità di negoziazione di valori, bisogni e interessi divergenti, e di gestione dei conflitti in un contesto democratico. Nel sistema scolastico italiano, ad esempio, l'insegnamento dell'educazione civica costituisce uno spazio importante per questo tipo di pratiche educative, così come l'introduzione di servizi di mediazione nelle scuole e offerte formative sulla comunicazione nonviolenta e la gestione efficace dei conflitti per tutti i componenti del sistema scuola - dagli alunni, agli insegnanti, al personale tecnico amministrativo, dirigenti scolastici e genitori (Panerai, Baukloh, 2014).

All'interno della famiglia l'insegnamento delle pratiche di cittadinanza attiva dovrebbe comprendere, oltre all'insegnamento dei diritti e dei doveri, lo sviluppo delle competenze che gli individui possono utilizzare per esprimere il proprio consenso o la loro opposizione nelle interazioni sociali di cui sono parte. Questo potrà far sì che l'educazione familiare diventi una scuola per la cittadinanza attiva e per relazioni umane senza violenza - e non un elemento che contribuisce alla radicalizzazione e allo sviluppo di comportamenti antisociali.

Saper gestire i conflitti in modo costruttivo è una capacità chiave non solo per il benessere relazionale in famiglia, ma anche per la partecipazione democratica nella società, consentendo l'autoaffermazione e la protesta - ad esempio in reazione ad atti di discriminazione o razzismo - senza il rischio di innescare una reazione a catena di radicalizzazione, comportamenti antisociali e violenza. La capacità personale di gestire il conflitto costituisce quindi un insieme di 
competenze e atteggiamenti di grande importanza. Essa può essere insegnata in ogni contesto di socializzazione, in particolare nella scuola (Panerai, Baukloh, 2014) e nel contesto familiare (Novara, 2013).

Friedrich Glasl ha introdotto il concetto di conflict capability, la capacità di affrontare il conflitto in modo costruttivo, basata sui principi dell'assertività, del potenziale di arricchimento delle divergenze tra le parti, della possibilità di incanalare l'energia potenzialmente distruttiva del conflitto in direzioni costruttive (1997, trad. it. 2019). Questa capacità personale può a sua volta contribuire a rendere più resilienti a tensioni e conflitti le organizzazioni e le strutture sociali (ibidem).

Il concetto di capability, o capacità individuale, è stato reso popolare dagli studi di Amartya Sen (1999, trad. it. 2000) e Martha Nussbaum (2011, trad. it. 2012) sullo sviluppo umano, come strumento per una valutazione comparativa dello sviluppo umano, e come fondamento per una teoria della giustizia sociale. Alla base del concetto è la domanda: cosa è in grado di fare ed essere ogni persona, in un determinato contesto istituzionale e sociale (Nussbaum, 2011, trad. it. 2012). A nostro avviso, la capacità di affrontare in maniera costruttiva i conflitti in cui si è coinvolti costituisce a pieno titolo una capacità interna agli individui, nel senso elaborato da Nussbaum, che deve trovare riscontro in appropriati canali istituzionali per la sua promozione e realizzazione effettiva nella società, per diventare quella che l'autrice chiama capacità combinate (Ibidem). Come per le capabilities nel discorso di Nussbaum (Ibidem), la capacità di affrontare il conflitto in modo costruttivo prescinde dai valori e dagli obiettivi dei contendenti, e non costituisce un rigido parametro normativo imponendo obblighi di comportamento: piuttosto, traccia una pluralità di percorsi di azione che gli individui possono intraprendere, e si riconnette alla capacità di pensiero critico, una delle capabilities fondative individuate dalla filosofa statunitense.

Nelle prossime pagine presentiamo alcune dimensioni della conflict capability, che riteniamo di immediata rilevanza per i contesti familiari e per rafforzare il ruolo delle donne nella prevenzione della radicalizzazione e dei comportamenti antisociali: la capacità di comprendere e interrompere le dinamiche di conflitto grazie ai modelli di analisi e intervento nei processi di escalation; la capacità di esprimere i propri interessi, esigenze, emozioni e valori e di ascoltare gli altri, anche se le posizioni assunte sono diametralmente opposte; la capacità di analizzare i propri sistemi di riferimento (famiglia, scuola, società) per affrontare i conflitti e riconoscere le potenzialità di cambiamento.

Tutte queste competenze contribuiscono allo sviluppo della capacità dei singoli di affrontare conflitti e al miglioramento qualitativo delle interazioni nei sistemi sociali di cui fanno parte, come la famiglia o la scuola. Educatori, insegnanti, operatori sociali e qualsiasi altra persona di riferimento che abbia acquisito delle competenze in materia di conflitti, possono svolgere un ruolo di sostegno e contribuire in questo modo a migliorare le relazioni interpersonali e a prevenire la radicalizzazione.

È quindi plausibile aspettarsi che nei contesti familiari l'aumento delle capacità di gestione nonviolenta dei conflitti potrà favorire l'assunzione di un ruolo attivo da parte delle figure di riferimento, e in particolare delle madri, nella prevenzione degli estremismi violenti, e lo sviluppo di nuovi role models per i membri della famiglia: un aspetto da non sottovalutare in un'ottica di genere.

\section{Comprendere e interrompere le dinamiche di escalation conflittuale}

La comprensione dei processi di escalation conflittuale consente un intervento appropriato e tempestivo. Qui presentiamo il modello analitico del mediatore austriaco Friedrich Glasl (1997, trad. it. 2019). Già all'inizio degli anni ' 80 , Glasl ha sviluppato un modello sofisticato per una 
migliore comprensione dei processi di escalation dei conflitti sociali, che è stato successivamente utilizzato dai mediatori di tutto il mondo per la prevenzione e l'intervento nei conflitti sociali. Friedrich Glasl differenzia anzitutto fra divergenze e conflitti: «Le divergenze sono la cosa più naturale del mondo nella vita delle persone; ma esse di per sé non costituiscono ancora un conflitto. Tutto dipende dal modo in cui le divergenze si gestiscono. La tensione diventa conflitto quando le persone coinvolte non riescono più a gestirla in modo costruttivo» (ivi, p. 123).

Nel suo modello d'analisi della dinamica dell'escalation, che gradualmente portano alla radicalizzazione del conflitto e al reciproco rafforzamento di aggressività e impulsi distruttivi, Glasl distingue nove livelli articolati in tre fasi principali, durante i quali le parti in conflitto perdono passo per passo l'autocontrollo e il controllo della situazione (Ibidem). Questa dinamica complessiva è illustrata nel grafico che segue.

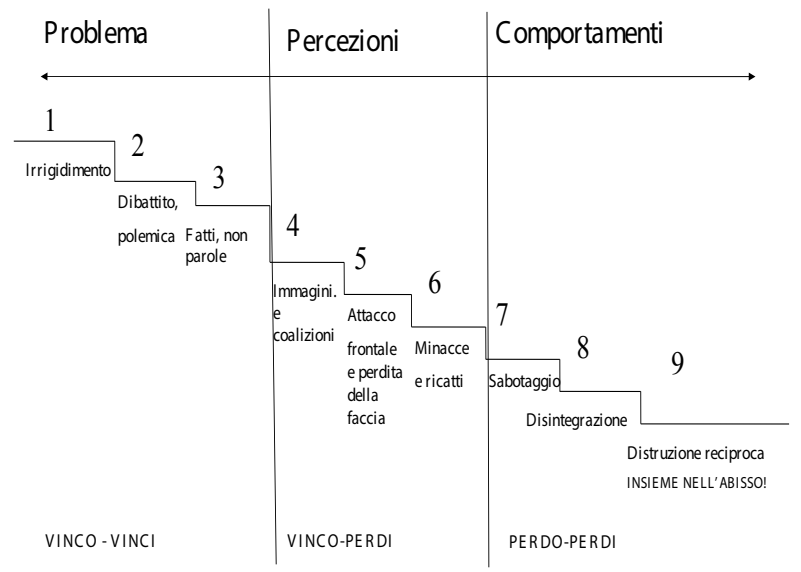

Grafico 1 - Dinamica dell'escalation conflittuale ${ }^{2}$

Il grafico riassume i gradini che portano verso la distruzione reciproca come risultato ultimo, sel'escalation conflittuale non viene interrotta. Illustriamo brevemente le caratteristiche principali e la loro rappresentazione nel grafico: le due linee verticali indicano il passaggio da una fase all'altra, e sull'asse orizzontale, le parole problema, percezioni, comportamenti riassumono le caratteristiche specifiche delle tre fasi nell' escalation conflittuale.

Il grafico può essere letto sia nella direzione dell'escalation conflittuale - verso il basso -, sia a ritroso per descrivere il processo di de-escalation durante la mediazione dei conflitti: tornando dall'ultima fase (comportamenti), nella quale le parti si concentrano soltanto sui comportamenti, percepiti esclusivamente come distruttivi, verso la seconda (percezioni), nella quale le parti lottano per costruire una loro immagine positiva e un'immagine esclusivamente negativa della controparte fino a risalire ai gradini della prima fase (problema) dell'escalation conflittuale.

All'inizio dell'escalation conflittuale le parti discutono di un problema, una divergenza che non riescono a risolvere. Quando passano dalle parole ai fatti (gradino tre) la qualità dell'interazione

${ }^{2}$ Cfr. Glasl, 1997, trad. it. 2019, in particolare le pp. 124-125; adattato dall'Autrice, N.d.A. 
peggiora notevolmente e le parti abbandonano l'idea iniziale, che da questo conflitto possono uscire entrambi vincitori (indicato nel grafico con le parole vinco-vinci). Nella fase due e tre la percezione riguardo ai possibili esiti del conflitto peggiora, da vinco-perdi (fase due) a perdo-perdi (fase tre), quando le parti hanno ben chiaro che nessuno dei due uscirà vincitore e fare soffrire l'altro, danneggiare di più l'altro che venir danneggiato da lui o lei, diventa la misura di successo per le parti; si scatena una dinamica distruttiva che può portare alla distruzione reciproca, se nessuno interviene, separando le parti con forza o se le parti si bloccano da sole ad esempio a causa dei rimorsi morali o della paura delle conseguenze penali.

Prima di analizzare la dinamica dell'escalation conflittuale, chiediamo ai lettori e alle lettrici di prendersi un momento per pensare ad una situazione conflittuale che hanno vissuto in prima persona e provare a rispondere alle domande: che cosa è successo? Come vi siete sentitile? Che cosa è cambiato durante l'escalation conflittuale? Come è finita l'escalation conflittuale? La soluzione del vostro conflitto aveva a che fare qualcosa con il problema iniziale o il conflitto si era spostato verso altri temi, o aveva coinvolto altre persone? Come vi sentite a pensare ora a questo conflitto e al vostro vissuto? C’è qualcosa che faresti diversamente?

Queste domande ci servono per sollecitare delle immagini, emozioni e riflessioni, che fanno sì che l'analisi dell'escalation non rimanga un esercizio astratto, ma contribuisca alla capacità di meta-analisi, alla conflict capability, prendendo atto delle emozioni proprie e assumendosi la responsabilità delle proprie azioni e dei loro effetti sulla nostra controparte e l'ambiente circonstante (famiglia, scuola, contesto lavorativo). Quando siamo dentro una dinamica di escalation spesso le nostre azioni, anche quelle più estreme, ci sembrano giustificate. Riusciamo a riconoscere il nostro contributo all'escalation conflittuale, la dissonanza fra le nostre azioni e i nostri valori di riferimento, soltanto quando ci liberiamo dalla rete distruttiva di percezioni distorte, emozioni alterate e (re)azioni sproporzionate, che ci sembravano senza alternativa, ma che ci lasciano perplessi, e a volte anche imbarazzati, quando le riconsideriamo a mente fredda.

Glasl propone di chiederci se «sono io ad avere un conflitto o è il conflitto a controllare me?» (1997, trad. it. 2019, p. 123). Con l'avanzare dell'escalation, le parti in conflitto perdono l'autocontrollo; più è profondo un certo livello, più è intensa e violenta la manifestazione del conflitto e la «forza di attrazione del meccanismo del conflitto spinge quest'ultimo verso il basso, a meno che gli avversari non si sveglino, contrastando questa dinamica interna. Tutto ciò richiede però da parte loro conoscenza dei meccanismi, capacità di percepire il processo in corso, consapevolezza e forza morale» (ivi, p. 125). La mediazione sistemica trasformativa vuole dare un contributo proprio in questo senso, dando alle persone degli strumenti per poter uscire da soli dalla dinamica di escalation (nella prima fase) o - se il conflitto è già entrato nella seconda fase dell'escalation (dal livello 4 in poi), chiamando un aiuto esterno professionale.

Qui non possiamo entrare nei dettagli dell'analisi e dei vari strumenti per interrompere l'escalation conflittuale e facilitare processi di cambiamento individuale e organizzativo (Ballreich, Glasl, 2019, trad. it. 2021; Baukloh, 2014; Glasl, 1997, trad. it. 2019; Panerai, Baukloh, 2014; Scotto, 2017). Illustreremo qui gli aspetti principali più rilevanti per il nostro tema. Per la prevenzione della radicalizzazione e dei comportamenti antisociali la conoscenza delle dinamiche di escalation è fondamentale, sia per riconoscere quando c'è bisogno di un intervento di una terza parte e per decidere che tipo di professionalità fare intervenire (dai mediatori, educatori, assistenti sociali o psicologi fino alle forze dell'ordine), sia per la scelta del metodo d'intervento: mentre nella prima fase dell'escalation le parti in conflitto sono ancora raggiungibili con argomentazioni e sono motivate ad affrontare direttamente il loro problema, a partire dalla seconda fase d'escalation, sono l'altra persona, l'altro gruppo o la società per intero a essere diventati "il problema”. Le questioni iniziali sono passate in secondo piano, l'emozioni sono 
alterate e il conflitto è diventato una battaglia che potrà vedere soltanto un vincitore (la logica del vinco-perdi). In questa fase del conflitto è molto probabile che la radicalizzazione interiore si esprima in comportamenti antisociali e che gli agenti di tali comportamenti li percepiscano come giustificati e senza alternativa.

Mano a mano che l'escalation va avanti, la distruttività aumenta, la capacità di autoriflessione diminuisce e le aspettative negative si rafforzano, trascinando le parti in una corsa verso il basso.

La ricerca di alleati (seguendo il motto "o sei con me, o sei contro di me"), lascia sempre meno spazio a intermediari, aumentando il numero di persone coinvolte nel conflitto e i temi conflittuali (livello 4); con azioni che mirano a far perdere la faccia alla controparte in pubblico o davanti ai suoi sostenitori, per "smascherare" colui che viene ormai percepito soltanto negativamente, mentre il proprio gruppo di sostenitori viene visto come impeccabile, vittima delle aggressioni degli altri, e portatore di valori e principi giusti, minacciati dalla controparte (livello 5). Questa visione porta a una accelerazione dell'escalation, perché di fronte a un avversario così negativo, i "freni" morali non tengono più, e azioni che prima non sembravano pensabili e giustificabili, ora sembrano alle parti in conflitto l'unica strada percorribile per difendere sé stessi, i propri obiettivi e valori.

L'escalation entra così nell'ultimo livello della fase vinco-perdi (livello G), con minacce e contro-minacce che accelerano ulteriormente l'escalation, vincolando i confliggenti alle azioni negative minacciate, con un aumento notevole del livello dello stress, ultimatum e contro-ultimatum, che portano alla terza fase (perdo-perdi). Da questo momento fare del male al nemico diventa la priorità, e si passa dall'obiettivo della distruzione limitata (livello 7 ) a quella illimitata (livello 8) fino alla ultima fase d'escalation (livello 9) con l'obiettivo dell'annientamento del nemico a costo dell'autoannientamento (Glasl, 1997, trad. it. 2019).

Anche se questa descrizione sembra un racconto estremizzato di un processo di radicalizzazione, dobbiamo sottolineare che è possibile incontrare questa dinamica di escalation conflittuale anche nei conflitti di vicinato, nelle aziende, nelle situazioni di mobbing sul posto di lavoro o a scuola, nella Rete, con i discorsi di odio o nei contesti familiari. Per poter intervenire o per prevenire queste dinamiche è importante essere consapevoli che l'escalation conflittuale ha un impatto su tutti gli attori nel sistema e - se non c'è una formazione adeguata e la consapevolezza di queste dinamiche - l'escalation conflittuale limita notevolmente la capacità di reagire in modo adeguato. L'escalation conflittuale può avvenire anche con atti di violenza "fredda", come punizioni sproporzionate comunicate con un linguaggio burocratico, ma non per questo meno violente $\mathrm{o}$ - se pensiamo alla scuola - con la ridicolizzazione del confliggente, ad esempio dell'alunno, davanti alla classe.

Perciò allenare la propria conflict capability e non farsi catturare dalla dinamica dell'escalation risulta fondamentale per tutti coloro che intendono dare un contributo costruttivo per la prevenzione della radicalizzazione e dei comportamenti antisociali. Prima di illustrare alcuni strumenti concreti di intervento proponiamo un ulteriore elemento per l'analisi dell'escalation conflittuale - la dinamica interiore.

\section{La dinamica di escalation interiore}

Abbiamo visto che non è facile resistere alla spirale di escalation. Per poter interrompere l'escalation conflittuale è necessario capire meglio la dinamica d'escalation interiore. Che cosa succede alla nostra capacità di percepire con accuratezza ciò che accade intorno a noi, quando siamo coinvolti in una interazione conflittuale? Come cambia il nostro stato d'animo? Quali sono le nostre emozioni e che cosa vogliamo raggiungere attraverso le nostre azioni? Friedrich Glasl 
ha osservato, nella sua lunga esperienza da mediatore dei conflitti, che l'escalation conflittuale ha un forte impatto distorsivo sulla nostra lucidità, la nostra capacità di avere una idea accurata di quello che succede intorno a noi e in noi stessi. La nostra capacità di ragionare diminuisce, non abbiamo più un quadro complessivo delle nostre emozioni, dei nostri bisogni, e spesso le nostre azioni non sono alienati con i nostri sentimenti e desideri più profondi, ma sono l'effetto di una dinamica quasi automatica fra stimoli esterni e risposte nostre, perdendo sempre di più di vista l'appropriatezza delle nostre azioni.

Nel libro Auto-Aiuto nei Conflitti (1997, trad. it. 2019), Glasl analizza in dettaglio cause ed effetti dei mutamenti delle funzioni psichiche, che possiamo caratterizzare qui soltanto in estrema sintesi, differenziando fra:

mutamenti della percezione - si formano immagini della realtà differenti, tuttavia ogni persona crede di avere l'immagine corretta e che sia l'altra parte a distorcere la realtà (ivi, p. 51);

mutamenti della rappresentazione, della memoria, del pensiero e dell'interpretazione-emergono sempre più semplificazioni nel pensiero degli individui, oltre a generalizzazioni, approssimazioni, immagini in "bianco e nero", concetti e rappresentazioni polarizzate (ivi, p. 52);

mutamenti della vita emotiva - alla «base di tutte le deformazioni delle funzioni psichiche ci sono tensioni e stress, [...] una suscettibilità accentuata che alimenta una sfiducia incipiente» e rafforza l'insicurezza. Le parti del conflitto non sopportano più di «provare sentimenti allo stesso tempo positivi e negativi di fronte alle cose, agli atteggiamenti e alle azioni dell'avversario» (ivi, p. 54).

Questa ambivalenza viene sciolta riservando i sentimenti positivi per sé e per la propria parte, e quelli negativi per l'avversario in modo da rendere univoca la situazione emotiva, perdendo gradualmente l'empatia reciproca, la capacità di mettersi nei panni altrui, isolandosi emotivamente gli uni dagli altri, diventando prigionieri della propria disposizione d'animo, della ripetizione continua delle stesse idee e dell' isolamento affettivo: "Ci chiudiamo nel bozzolo del nostro mondo interiore e così finiamo con il perdere noi stessi, perché è solo nel dialogo con il mondo esterno che possiamo realizzare il nostro Sé» (ivi, p. 54-55);

mutamenti della volontà - irrigidimenti, a causa delle delusioni vissute, focalizzazione su pochi obiettivi, da realizzare a ogni costo; «la volontà si restringe a poche possibilità, si fa assoluta e radicale. Col tempo si diffonde il fanatismo. Prima del conflitto eravamo in grado di riconoscere numerose alternative della volontà e dell'azione per soddisfare i nostri bisogni in modi diversi. Con il conflitto, ci ritroviamo a perseguire pochissimi bisogni. Invece di esprimere i nostri veri bisogni, ci ritroviamo a formulare solo posizioni e pretese» (ivi, p. 55);

mutamenti del comportamento esteriore - il comportamento perde la sua varietà, diventa sempre più povero e monotono. «Le parole e le azioni coincidono solo in parte con le intenzioni. In questa maniera si manifestano sulle altre persone vari effetti non intenzionali e che non sono nemmeno percepiti dall'agente» (ivi, p. 56).

Questi effetti indesiderati possono avere conseguenze anche gravi sulla controparte, senza che qualcuno se ne assuma la responsabilità.

Perciò emergono pericolose zone demonizzate: il mio avversario vive a causa mia esperienze spiacevoli che però non corrispondono alle mie intenzioni. Egli reagisce all'attacco e mi provoca a sua volta 
esperienze che non coincidono con le sue intenzioni. Ma intanto gli effetti ci sono già da entrambe le parti, intenzionali o meno. Le parti del conflitto si incolpano a vicenda - e nessuna delle due è disposta ad assumersi la responsabilità delle conseguenze indesiderate. In questo modo i conflitti si inaspriscono ulteriormente. Nell'elaborazione dei conflitti si tratterà di considerare attentamente il distacco fra intenzione, comportamento ed effetto (ivi, pp. 56-57).

Porsi la domanda "sono io che ho un conflitto, o è il conflitto che controlla me?" è un primo passo per riconquistare l'autonomia. Un ulteriore passo è quello di abbandonare l'idea di una linearità nell'escalation conflittuale, la "ricerca del colpevole", perché come abbiamo visto nell'analisi dell'escalation esteriore e interiore, nei conflitti c'è sempre una causalità reciproca, che viene chiamata nel linguaggio della teoria dei sistemi "causalità circolare" (Ibidem, passim). Nella mediazione dei conflitti questo passaggio - abbandonare la ricerca del colpevole e analizzare insieme la dinamica di escalation adottando la prospettiva della causalità circolare - risulta spesso molto difficile per le parti in conflitto. Però, una volta intrapresa questa strada, l'orizzonte si apre e le parti possono riuscire a lavorare insieme alla soluzione del loro conflitto. Per la prevenzione della radicalizzazione e dei comportamenti antisociali l'analisi della dinamica interiore dell'escalation diventa fondamentale, perché ci fa capire gli ostacoli per la comunicazione con una persona che vive il conflitto, e che non riusciamo a raggiungere con delle argomentazioni, appelli emotivi, divieti o minacce, senza contribuire a nostra volta all'escalation. Più tempo passa, più difficile sarà l'interazione comunicativa.

C'è bisogno di un approccio che faciliti la comprensione e permetta di instaurare una relazione con l'altro al di fuori degli schemi dell'interazione conflittuale. Chiara Biasin, Vanna Boffo e Clara Silva sottolineano l'importanza della dimensione comunicativa nei contesti educativi e formativi, «dove va sempre tenuto conto che la dimensione comunicativa non implica solo l'espressione di un pensiero ma sollecita anche la cura nella scelta della modalità appropriata con cui esprimere tale contenuto di pensiero (scelta delle parole, chiarezza e semplicità dell'espressione, attenzione agli aspetti non verbali ecc.) (2020, p. 11)».

L'attenzione ad alcuni principi di base nell'interazione comunicativa, l'ascolto e la comunicazione autentica delle proprie emozioni sono stati ulteriormente sviluppati da vari Autori (ad esempio Schulz von Thun, 1999, trad. it. 2002; Sclavi, 2003), e fanno parte della educazione alla nonviolenza (Galtung, 1996, trad. it. 2000; L'Abate, 1985; Patfoort, 1992, trad. it. 2000; Rosenberg, 1998, trad. it. 2003) e della formazione alla mediazione dei conflitti (Baukloh, 2014).

Nei contesti familiari i genitori - e specialmente le madri, se sono loro ad avere il ruolo primario di educazione e presenza quotidiana - hanno un compito assai difficile: instaurare una interazione comunicativa efficace con un individuo giovane "catturato" dalla dinamica di escalation interiore, che compromette la sua capacità di cogliere l'intenzione costruttiva dei genitori e rischia di rafforzare la percezione di non comprensione e la tendenza alla chiusura.

In questa prospettiva sono utili i suggerimenti dello psicologo statunitense Thomas Gordon per il superamento delle barriere comunicative e lo sviluppo di una genitorialità efficace, in grado di prevenire e contrastare comportamenti antisociali e la perdita della possibilità di comunicazione con i propri figli. Partendo dai lavori di Carl Rogers (1977, trad. it. 1978), Gordon elabora, già negli anni '70, un approccio educativo basato sull'ascolto e sui principi della nonviolenza. Nei suoi scritti sulla comunicazione efficace, Gordon evidenzia una serie di barriere comunicative, che vanno da quelli più immediatamente riconoscibili come minacciare, rimproverare, giudicare, ridicolizzare, minimizzare, ironizzare fino alle pratiche comunicative che spesso non vengono sospettate di poter avere degli effetti negativi sull'interazione comunicativa, come ad esempio offrire soluzioni e consigli o interpretare (2002, trad. it. 2014). Come metodo di superamento di queste barriere, Gordon propone uno stile comunicativo diverso, 
applicabile in vari ambiti, dalla scuola (1970a, trad. it. 1991) alla leadership efficace (1977, trad. it. 2016) come nella vita di tutti i giorni e nei contesti familiari (1970b, trad. it. 1997), illustrati nelle prossime pagine.

\section{Thomas Gordon: diventare genitori efficaci e educare figli responsabili}

«Tutti incolpano i genitori dei problemi dei giovani [...]. Ma chi aiuta i genitori?» si chiede Thomas Gordon nel suo libro Genitori efficaci. Educare figli responsabili, uscito negli Stati Uniti nel 1970 (trad. it. 1997, p. 15). L'approccio all'educazione di Gordon mira alla capacitazione dei singoli e del sistema educativo, e apre un orizzonte di speranza per genitori e educatori, quando scrive: «Oggi sono convinto che gli adolescenti non si ribellano ai genitori. Si ribellano solamente ad alcuni metodi disciplinari distruttivi adottati quasi universalmente dai genitori. Il dissenso e la turbolenza emotiva sono un'eccezione, non la regola, quando i genitori imparano un nuovo metodo per risolvere i conflitti» (Ibidem).

Partendo dall'idea che l'accettazione dell'altro così come è forma la base di una relazione educativa sana, Gordon spezza una lancia per una interazione comunicativa autentica, sincera: ciò include anche la comunicazione della non accettazione di alcuni comportamenti dei propri figli, mettendo dei limiti precisi, senza tuttavia svalorizzare la persona e evitando di intervenire in situazioni che l'altro potrebbe anche risolvere da solo. Il rispetto dell'autonomia dei figli - riuscire ad accettare che i figli sono individui con una propria vita, una propria identità e mostrare comprensione ed empatia per i loro sentimenti senza sottrarle la responsabilità del problema - è fondamentale per una comunicazione efficace e l'educazione di figli responsabili (1970b, trad. it. 1997).

Gordon definisce l'interruzione della relazione fra figli e genitori come "licenziamento" dei genitori: «Questi vengono licenziati dai figli dopo averli assillati e tediati con lunghi sproloqui per riuscire a modificare comportamenti e valori tenuti in alta considerazione dai figli. Gli adolescenti congedano i propri genitori quando avvertono che essi tentano di negare loro diritti civili fondamentali» (ivi, p. 143). Cercando di modellare i propri figli affinché somiglino agli adulti e agiscano secondo ciò che gli adulti ritengono essere giusto o sbagliato, i genitori perdono la propria influenza. Per poter avere un ruolo costruttivo nella prevenzione di comportamenti antisociali dei propri figli, i genitori si dovrebbero limitare a cercare di modificare soltanto quei comportamenti dei figli che incidono concretamente e tangibilmente sulla propria esistenza. Gordon afferma: «la mia esperienza con ragazzi di tutte le età mi ha insegnato che in genere essi sono assai disponibili a modificare un comportamento quando si accorgono che veramente impedisce a qualcun altro di soddisfare i propri bisogni» (ivi, p. 145).

Questa affermazione vale per l'educazione come per la mediazione dei conflitti: distinguere fra ciò che mi disturba nel comportamento dell'altro, ma non ha un impatto diretto su di me, e ciò che vorrei chiedere alla mia controparte di cambiare perché mi provoca sofferenza, è un primo passo importantissimo nella gestione efficace dei conflitti sociali, che purtroppo spesso le persone coinvolte nel conflitto hanno difficoltà a fare. Ad esempio se nel conflitto fra vicini una parte si lamenta del "cattivo odore" che proviene dalla cucina dei vicini, aggiungendo "non so proprio come fanno a mangiare queste schifezze", la probabilità che i vicini siano disponibili ad ascoltare e a cambiare il loro comportamento è molto bassa. Viceversa, il messaggio "non riesco più a stendere i panni davanti casa mia perché l'odore che proviene dalla vostra cucina impregna i miei panni appena lavati" è una descrizione dell' effetto del comportamento del vicino e può portare a una ricerca di soluzioni soddisfacenti per tutti, come avviene spesso nei servizi di mediazione dei conflitti di vicinato (Baukloh, Valdambrini, 2015). 
Thomas Gordon ha chiamato il suo metodo per la soluzione dei conflitti «problem solving senza perdenti» (1970a e b, trad. it. 1997, passim), articolandolo in sei fasi, che vanno dalla identificazione e definizione del conflitto (fase 1), alla ricerca creativa di soluzioni (fase 2), alla valutazione delle soluzioni emerse (fase 3), alla scelta della soluzione migliore (fase 4), alla programmazione e attuazione della soluzione ( fase 5), fino alla verifica dei risultati nella fase 6 (ivi, p. 128). Gli elementi centrali del problem solving senza perdenti - l'ascolto attivo, il messaggio in prima persona, la ricerca di soluzioni accettabili per entrambi - sono gli stessi di tanti altri approcci alla mediazione dei conflitti, che si sono in parte ispirati a Thomas Gordon e si sono sviluppati e diffusi in vari settori della società.

\section{Il processo di mediazione dei conflitti}

Esistono molti approcci diversi all'intervento di una terza parte in un conflitto in funzione mediativa. Per gli scopi di questo articolo non è fondamentale distinguere i vari approcci alla mediazione e alla gestione del colloquio, ma è opportuno focalizzare l'attenzione sui meccanismi di base, che fanno sì che nell'incontro di mediazione le parti in conflitto interrompano la loro interazione conflittuale e prendano in mano la situazione, trasformando la loro relazione con l'aiuto della mediatrice o del mediatore.

In un approccio sistemico trasformativo alla mediazione, questi elementi vengono inseriti in una cornice che permette di analizzare l'interazione conflittuale all'interno del rispettivo sistema di riferimento - famiglia, scuola, organizzazione, società (Ballreich, Glasl, 2019, trad. it. 2021; Baukloh, 2014; Baukloh, Scotto, 2019; Glasl, 1997, trad. it. 2019; Scotto, 2017) - e lavora in modo fluido, senza seguire uno schema in fasi, ma seguendo in modo proattivo la dinamica dell'interazione conflittuale delle parti, facilitando processi di cambiamento attraverso la capacitazione del singolo, di riconoscere i propri bisogni ed emozioni e di instaurare una relazione comunicativa basata sul riconoscimento dei bisogni ed emozioni della controparte (Bush, Folger, 2004, trad. it. 2009; Mosca, 2018).

Proviamo adesso a caratterizzare brevemente il processo di cambiamento che avviene all'interno dell'incontro di mediazione - o in generale all'interno di processi di cambiamento nelle organizzazioni o nei gruppi facilitati con un approccio trasformativo -, utilizzando una immagine assai diffusa: l'esplorazione della profondità del conflitto e della possibilità di un futuro migliore con una cosiddetta "procedura a U” (Baukloh, Scotto, 2019; Glasl, 1997, trad. it. 2019; Scharmer, 2009, trad. it. 2018; Senge, 2006, trad. it. 2019; Senge et al., 2005, trad. it. 2013).

La procedura a U inizia nella mediazione sistemica trasformativa con la ricognizione

U del conflitto come rappresentato dalle parti: l'intenzione comunicativa, i temi conflittuali, la tipologia del conflitto (su una questione specifica, sulla difesa o conquista di una posizione diversa nella relazione o sul cambiamento del sistema

sociale o organizzativo a cui i contendenti appartengono), le persone coinvolte e le loro relazioni formali e informali, il livello d'escalation e l'atteggiamento delle parti in rispetto al conflitto.

A partire da queste informazioni si realizza una prima mappatura del conflitto, fatta insieme alle parti attraverso domande aperte, non direttive e una modalità particolare di ascolto da parte dei mediatori, spesso chiamata "ascolto attivo", o "ascolto a 360 gradi”, perché basato sull'accoglienza delle parti e l'accettazione di tutto quello che loro esprimono, senza formulare giudizi e con un massimo di concentrazione verso tutto ciò che viene espresso sia in modo verbale che non verbale.

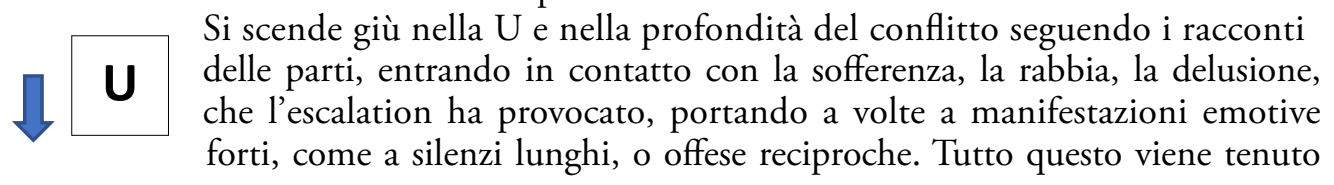


insieme dalla mediatrice in un "contenitore" costruttivo, attraverso domande di approfondimento, il rispecchiamento delle emozioni espresse verbalmente e non verbalmente, il reframing (l'espressione dei concetti espressi con le parole del mediatore) e il riassunto da parte della mediatrice stessa. Quest'ultimo strumento si rivela di grande importanza, perché il riassunto da parte del mediatore di ciò che è stato appena detto da una parte fa sì che le parti in conflitto riescano ad ascoltare con maggiore facilità quello che l'altro esprime - a volte per la prima volta dopo molto tempo di incomprensioni reciproche - e a entrare in contatto con le proprie emozioni, riconoscendo le distorsioni che il loro modo di vedere se stessi e l'altro ha subito.

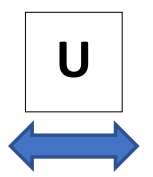
Durante questo processo comunicativo le parti acquisiscono passo per passo una maggiore autoconsapevolezza, riacquistano il controllo di sé stesse e una visione dell'interazione conflittuale al di fuori delle deformazioni percettive e idee idealizzate di sé stessi e demonizzate della contro parte. Si tratta di un processo di capacitazione e riconoscimento, che inizia singolarmente e diventa condiviso nel momento in cui la controparte non viene più vista come nemico, ostacolo principale per il raggiungimento dei miei obiettivi o minaccia per la mia esistenza, ma viene nuovamente vista come possibile partner per la soluzione del problema iniziale.

Con il supporto del mediatore l'interazione comunicativa cambia, ad esempio attraverso l'utilizzo di messaggi in prima persona: invece di focalizzare il messaggio sull'altra persona e le interpretazioni e giudizi in merito all'altro (messaggi-tu), il mediatore invita le parti a descrivere la situazione nel modo più concreto possibile, di dire alla controparte come si sono sentiti, che cosa hanno provato e perché e che cosa vorrebbero che succedesse in futuro in una situazione simile (messaggio-io). L'empatia e la capacità di vedere la situazione dal punto di vista altrui aumentano e portano le parti ad una maggiore comprensione delle emozioni e dei bisogni della contro-parte e gradualmente ad una apertura emotiva.

Arriva quindi il momento per parlare delle regole scritte e non scritte che fino ad oggi hanno regolato la relazione delle parti e di identificare insieme, se c'è bisogno di cambiare qualcosa - nella relazione fra le parti e nei rispettivi sistemi di riferimento. "Come vogliamo vivere la nostra relazione in futuro?" e "di che cosa abbiamo bisogno per arrivare a questo futuro?", sono le domande che le parti si pongono ora. La mediatrice facilita questo dialogo sul presente e sul futuro desiderato. Sono le parti stesse a determinare quanto andare in profondità nell'analisi e nell'entità dei cambiamenti desiderati.

La mediazione procede con la "risalita" nella procedura U, portando le parti U a parlare delle soluzioni per i problemi identificati da loro, a ragionare su un piano d'azione e possibili ostacoli nella sua implementazione e a concordare meccanismi per la verifica e la gestione alternativa dei problemi nel caso che l'accordo non dovesse funzionare; senza ricorrere nuovamente all' escalation conflittuale - quello che nel processo del problem solving senza perdenti di Gordon illustrato in precedenza sono le fasi 2-5. Se le parti lo desiderano possono mettere il loro accordo per iscritto. Nel caso si tratti di una mediazione formalizzata (come ad esempio la mediazione civile e commerciale in Italia), ci sono da seguire le regole previste per la validazione dell'accordo concordato.

Che cosa è cambiato? Se pensiamo alla dinamica di escalation, possiamo dire che non è più il conflitto che controlla le parti, ma sono le parti che hanno riacquisito il controllo di sé stesse e della loro interazione. Non è determinante se il conflitto sia stato completamente risolto nel corso della mediazione. Potrebbe accadere che ci vuole più tempo per affrontare tutti i temi, che sia necessario coinvolgere più attori per poter risolvere la situazione in modo sodisfacente e duraturo. Potrebbe anche essere che le parti si sono rese conto che non intendono continuare la loro relazione e che per loro la soluzione del conflitto sta nella separazione; una separazione 
che però ora possono affrontare con più serenità e senza di cadere nuovamente nella trappola dell'escalation conflittuale.

\section{Prevenzione della radicalizzazione mediazione dei conflitti e facilitazione di processi di cambiamento}

Acquisire la capacità di gestire conflitti con la mediazione è un elemento fondamentale per la prevenzione della radicalizzazione e dei comportamenti antisociali e può avvenire sia attraverso corsi di formazione (ad esempio all'interno delle scuole; Panerai, Baukloh 2014) sia attraverso servizi di mediazione sociale ed interculturale, ad esempio all'interno della pubblica amministrazione (Baukloh, 2012a, 2018) o attraverso progetti della società civile (Baukloh, Scotto, 2015), servizi di consulenza per conflitti di vicinato, ad esempio nell'edilizia sociale (Baukloh, 2012b), o la mediazione familiare (Urso, 2012, 2013). Per fare sì che la mediazione sviluppi il suo pieno potenziale per la gestione costruttiva dei conflitti sociali, scolastici e familiari ci sarebbe però bisogno di un drastico aumento delle attività formative, partendo ad esempio dall'inserimento della formazione alla mediazione nei curricula delle professioni d'aiuto, del sistema educativo e delle pubbliche amministrazioni (Council of Europe, 2011).

Per le donne - sia nel ruolo di madri, sorelle o figlie all'interno del contesto familiare, sia al di fuori del sistema famiglia - la capacità di gestire in modo efficace i conflitti contribuisce all' aumento delle possibilità di fare sentire la propria voce, di sviluppare il proprio ruolo in modo allineato con i propri bisogno, emozioni e desideri e di avere un ruolo attivo nella società. Per il sistema famiglia questo significa diventare un sistema autoriflessivo, aperto al cambiamento e flessibile nella gestione delle sfide interne o esterne. Per i processi di cambiamento al livello della società, ciò significa facilitare una apertura verso altri punti di vista, nel senso del già citato "agonismo democratico" e la possibilità per tutti membri della comunità di partecipare alla vita pubblica.

Il riconoscimento reciproco, come illustrato nelle pagine precedenti dedicate al processo di mediazione, è fondamentale non soltanto per la gestione efficace dei conflitti nei contesti educativi o familiari, ma in generale per una convivenza pacifica nella società, basata sulla garanzia dei diritti e sull'accettazione degli individui indipendentemente dalle loro posizioni politiche, orientamenti sessuali o credenze religiose. Il filosofo Axel Honneth ha analizzato la centralità della lotta per il riconoscimento nei processi di cambiamento e gli effetti negativi per i singoli individui, i gruppi e la società in toto quando il riconoscimento viene negato (1995, trad. it. 2002).

Un importante presupposto per la riuscita dell'integrazione delle voci critiche, delle proteste e degli impulsi di cambiamento è indicato da Mouffe: «Il compito primo di una politica democratica non è quello di eliminare le passioni o relegarle nella sfera privata, così da stabilire un consenso razionale nella sfera pubblica. Piuttosto, è quello di "sublimare" le passioni, mobilitandole verso progetti democratici, creando forme collettive di identificazione intorno agli obiettivi democratici» (2000, trad. it. 2015, p. 29).

Ciò vale non solo per un sistema democratico sano, ma anche per un sistema familiare vitale, in cui i membri della famiglia sviluppano, nella vita di tutti i giorni, il rispetto per sé stessi e gli altri e la capacità di gestire conflitti in modo costruttivo. La realtà di molte famiglie è ben lontana da questo ideale, così come lo è la realtà di molte società democratiche. Gli approcci presentati, derivanti dalla mediazione sistemica trasformativa, possono contribuire a un processo di cambiamento che si traduca in un aumento dell'empowerment individuale, collegando la prevenzione della radicalizzazione con lo sviluppo di possibilità di identificazione positive e di partecipazione democratica.

Lynn Davies, pedagogista esperta in materia di educazione per la prevenzione della radicalizzazione e dell'estremismo violento, sottolinea nel suo libro Educating Against Extremism la 
necessità di una maggiore politicizzazione dei giovani attraverso l'educazione a riflettere e agire in modo critico, a partire dall'insegnamento nella scuola: un'educazione civica all'idealismo critico. «La risposta all'estremismo non è la moderazione, ma un idealismo altamente critico e informato» (Davis, 2008, p. 200)․․

\section{Conclusioni}

Rafforzare la conflict capability, la capacità di affrontare i conflitti da parte degli individui nei loro sistemi di riferimento con gli strumenti presentati nel presente articolo, consente di avviare processi di cambiamento sociale senza ricorrere alla violenza, e di promuovere la partecipazione sociale e politica, senza dover rinunciare ai propri valori, esigenze e interessi. Emozioni come la rabbia, provate in risposta a esperienze di ingiustizia, discriminazione o razzismo, o la sensazione di non appartenenza possono essere tramutate in energia trasformativa attraverso le competenze alla gestione del conflitto che abbiamo illustrato e sono in grado di fornire un rilevante contributo al cambiamento sociale.

Saper lottare per il proprio riconoscimento non è infatti soltanto una competenza salutare per il singolo, ma contribuisce alla qualità delle relazioni sociali e dell'intero sistema politico democratico, nella misura in cui le istituzioni sono in grado di fare propri i principi di una «democrazia agonistica», come definita da Mouffe (2000, trad. it. 2015, passim). La gestione costruttiva dei conflitti incoraggia a riesaminare le proprie percezioni e il proprio interesse al punto di vista dell'altro, la comunicazione nonviolenta e la decostruzione di stereotipi e pregiudizi alla base di scelte di azione inadeguate. In tal modo è possibile compiere i primi passi verso la responsabilizzazione, un impegno civico consapevole e la prevenzione di processi di radicalizzazione e comportamenti antisociali.

L'elaborazione degli Autori presentati fornisce numerosi strumenti in grado di far sì che la lotta per il riconoscimento non prenda una piega (auto)distruttiva, ma sia in grado di liberare energie costruttive per il cambiamento.

Il 20 gennaio 2021, in occasione della cerimonia di insediamento a Washington del nuovo Presidente americano Joe Biden e della Vicepresidente Kamala Harris, la giovane poetessa Amanda Gorman ha indicato con The Hill We Climb la strada verso una comunità democratica basata sull'equità, la nonviolenza e una conflittualità aperta e costruttiva. Vogliamo concludere questo scritto, riportando i suoi versi finali: «When day comes, we step out of the shade aflame and unafraid. The new dawn blooms as we free it. For there is always light. If only we're brave enough to see it. If only we're brave enough to be it» (Gorman, to published $)^{4}$.

\section{Riferimentibibliografici}

Ballreich R., Glasl F. (2019): La gestione sistemica dei conflitti nelle organizzazioni. Volume1: concetti, metodi ed esercizi per comprendere la dinamica interpersonale organizzativa dei conflitti (edizione italiana a cura di A.C. Baukloh e P. Lucarelli). Trad. it. Milanofiori - Assago: Wolters Kluwer, 2021.

Baukloh A.C. (2008): Il processo della mediazione". In S. Spinanti (a cura di): Impariamo a litigare. I quaderni di Janus. Roma: Zadig, pp. 91-110, 2008.

Baukloh A.C. (2014): La mediazione dei conflitti nell'ambito socio-sanitario: un modello per la formazione. In S. Lelli, F. Sacchetti, S. Tirini (a cura di): Conflitti identitari e pratiche delle istituzioni. Milano: FrancoAngeli, pp. 155-168.

Baukloh A.C. (2012a): Dialoghi interculturali - conflittualità, sfide e prospettive per la convivenza interculturale nella città di Prato. Prato/Firenze: Gambi.

\footnotetext{
${ }^{3}$ Traduzione a cura dell'Autrice, N.d.R.

${ }^{4}$ Per tutti i dettagli si rimanda ai Riferimenti bibliografici, N.d.R.
} 
Baukloh A.C. (2012b): I conflitti in ambito abitativo: inquadramento, casistica e buone prassi. In M.P. Ponticelli (a cura di): Linee guida per la gestione dei conflitti in ambito abitativo. Bolzano: Fondo Sociale Europeo/Prov. Autonoma di Bolzano/Alto Adige, pp. 5-14 (https://www.pin.unifi.it/images/ PINews/2021/03/fmarc/Linee_guida_per_la_gestione_dei_conflitti_in_ambito_abitativo_2012. pdf; data di ultima consultazione: 10.11.21).

Baukloh A.C. (2018): La mediazione di strada come catalizzatore del dialogo interculturale. Riflessioni sul progetto 'Giardini Comunicanti - Dialogo interculturale in aree verdi urbane' realizzato nella città di Prato. Prato: Laboratorio “Forma Mentis”, 2018.

Baukloh A.C., Scotto G. (2015): La mediazione dei conflitti e il progetto Empatic. In EMPowerment Associazioni e volon Tari Immigrati per la Comunità. PERCORSI E PRATICHE DI DIALOGO INTERCULTURALE. ANCI Umbria, pp. 34-55.

Baukloh A.C., Scotto G. (2019): Friedrich Glasl: microsociologia del conflitto, mediazione e sviluppo organizzativo. Massa: Lu:Ce Edizioni.

Baukloh A.C., Valdambrini A. (2015): La mediazione trasformativa. In P. Consorti, A. Valdambrini: Mediazione sociale. Riflessioni teoriche e buone pratiche. Pisa: Pisa University Press/Centro interdisciplinare Scienze per la Pace.

Biasin C., Boffo V., Silva C. (2020): Le dinamiche relazionali e comunicative nella formazione delle professionalità educative. RIEF-Rivista Italiana di Educazione Familiare, n. 2, pp. 7-25.

Bush R.A.B., Folger J.P. (2004): La promessa della mediazione. L'approccio trasformativo alla gestione dei conflitti. Trad. it. Firenze: Vallecchi, 2009.

Council of Europe (2011): Intercultural competences in Social Services. Constructing an Inclusive Institutional Culture. Strasbourg: Council of Europe Publishing.

Davies L. (2008): Educating Against Extremism. London: Trentham Books.

Davies L. (2009): Educating Against Extremism: Towards a Critical Politicisation of Young People. International Review of Education, n. 55, pp. 183-203.

Galtung J. (1996): Pace con mezzi pacifici. Trad. it. Milano: Esperia, 2000.

Glasl F. (1997): Auto-aiuto nei conflitti (edizione italiana a cura di A.C. Baukloh e G. Scotto). Trad. it. Firenze: Editpress, 2019.

Gordon T. (1970a): Insegnanti efficaci. Trad. it. Molfetta (BA): La Meridiana, 1991.

Gordon T. (1970b): Genitori efficaci. Educare figli responsabili. Trad. it. Molfetta (BA): La Meridiana, 1997.

Gordon T. (1977): Leader efficaci. Essere una guida responsabile favorendo la partecipazione. Trad. it. Molfetta (BA): La Meridiana, 2016.

Gordon T. (2002): Relazioni efficaci. Come costruirle. Come non pregindicarle. Molfetta (BA): La Meridiana, 2014.

Gorman A. (to be published): The Hill We Climb, Transcript of the Poem Delivered at the U.S. Presidential Inauguration after Joe Biden and Kamala Harris Were Sworn in as President and Vice President, 20.01.2021. Washington (https://thehill.com/homenews/news/535052-read-transcript-of-amanda-gormans-inaugural-poem; last access: 10.11.21).

Honneth A. (1995): La lotta per il riconoscimento. Trad. it. Milano: Il Saggiatore, 2002.

L'Abate A. (1985): Addestramento alla nonviolenza. Torino: Satyagraha Editrice.

Mosca C. (2018): La Mediazione Trasformativa. Treviso: TIAKI Publ. Editore.

Mouffe C. (2000): Il Conflitto Democratico (edizione italiana a cura di D. Tarizzo). Trad. it. Milano/Udine: Mimesis, 2015.

Novara D. (2013): Litigare fa bene. Insegnare ai propri figli a gestire i conflitti, per crescerli più sicuri e felici. Milano: Rizzoli.

Nussbaum M. (2011): Creare capacità. Liberarsi dalla dittatura del Pil. Trad. it. Bologna: il Mulino, 2012.

Panerai A., Baukloh A.C. (2014): Il Dialogo e la Mediazione dei Conflitti nella Scuola Interculturale. Manuale per insegnanti e formatori. Parma: Edizioni Junior.

Patfoort P. (1992): Costruire la nonviolenza.Per una pedagogia dei conflitti. Trad. it. Molfetta (BA): La Meridiana, 2000.

Ribolzi L. (2012): Società, persona e processi formativi. Milano: Mondadori Università.

Rogers C.R. (1977): Potere personale. La forza interiore e il suo effetto rivoluzionario. Trad. it. Roma: Astrolabio-Ubaldini, 1978. 
Rosenberg M.B. (1998): Le parole sono finestre (oppure muri). Introduzione alla comunicazione nonviolenta. Trad. it. Reggio Emilia: Esserci Edizioni, 2003.

Scharmer C.O. (2009): Teoria U. I fondamentali. Principi e applicazioni. Milano: Guerini Next, 2018. Schulz von Thun F. (1999): Parlare Insieme. Trad. it. Milano: TEA, 2002.

Sclavi M. (2003): Arte di ascoltare e mondi possibili. Milano: Bruno Mondadori.

Scotto G. (2017): Mediazione dei conflitti e salutogenesi: un modello sistemico-trasformativo. Riflessioni Sistemiche, n. 16, pp. 127-139.

Sen A. (1999): Lo sviluppo è libertà: perché non cề crescita senza democrazia. Trad. it. Milano: Mondadori, 2000.

Senge P. (2006): La quinta disciplina. L'arte e la pratica dell'apprendimento organizzativo. Trad. it. Napoli: Editoriale Scientifica, 2019.

Senge P., Scharmer O., Jaworski J., Flowers B.S. (2005): Presence. Esplorare il cambiamento profondo nelle persone, nelle organizzazioni e nella società. Trad. it. Milano: FrancoAngeli, 2013.

Urso E. (2012): La mediazione familiare: modelli, principi, obiettivi. Firenze: Firenze University Press.

Urso E. (a cura di) (2013): La mediazione familiare "oltre il conflitto": la comparazione giuridica all'interno di un percorso interdisciplinare. Firenze: Firenze University Press. 